\title{
Snail/FOXK1/Cyr61 Signaling Axis Regulates the Epithelial-Mesenchymal Transition and Metastasis in Colorectal Cancer
}

\author{
Xiaoting Huang ${ }^{\mathrm{a}}$ Li Xiang ${ }^{\mathrm{b}}$ Yueqiao Li $^{\mathrm{a}}$ Yingying Zhaoc Huiqiong Zhu ${ }^{\mathrm{a}}$ \\ Yizhi Xiao ${ }^{a}$ Mengwei Liu $^{a}$ Xiaosheng $W^{a}{ }^{a}$ Zhiqing Wang ${ }^{a}$ Ping Jiang ${ }^{a}$ \\ Haitao Qinga Qiang Zhang ${ }^{a}$ Guangnan Liua Wenjing Zhang ${ }^{\mathrm{d}}$ Aimin Lia \\ Ye Chen ${ }^{a}$ Side Liu, ${ }^{a, b}$ Jide Wang ${ }^{a, b}$ \\ aGuangdong Provincial Key Laboratory of Gastroenterology, Department of Gastroenterology, \\ Nanfang Hospital, Southern Medical University, Guangzhou, 'Department of Gastroenterology, \\ Longgang District People's Hospital, Shenzhen, 'Department of Gastroenterology, Panyu Central \\ Hospital, Guangzhou, 'Department of Medical Oncology, the First people's Hospital of Yunnan \\ Province, Medical School of Kunming University of Science and Technology, Kunming, China
}

\section{Key Words}

Snail $•$ FOXK1 $\bullet$ Cyr61 $\bullet$ Colorectal cancer $・$ Metastasis

\begin{abstract}
Background/Aims: Metastasis is the primary cause of colorectal cancer (CRC)-related death. However, the molecular mechanisms underlying metastasis in CRC remain unclear. Methods: We evaluated mRNA and protein expression levels by quantitative real-time reverse transcription $P C R$, western blotting, immunofluorescence, tissue microarrays, and immunohistochemistry assays. We also assessed the migration and invasion abilities of CRC cells in vitro by wound healing assays, invasion and migration assays, western blot analysis, and immunofluorescence. Tumor metastasis was evaluated in nude mice in vivo. Results: A positive correlation was observed between the expression patterns of Forkhead box k1 (FOXK1) and Snail in CRC. Luciferase reporter and chromatin immunoprecipitation assays demonstrated that Snail directly bound to and activated the human FOXK1 gene promoter. Moreover, the Snail-FOXK1 axis promote epithelial mesenchymal transition (EMT)-mediated CRC cell invasion and metastasis. FOXK1 and Snail expression levels were correlated with tumor progression and served as significant predictors of overall survival in patients with CRC. Furthermore, overexpression of FOXK1 induced the EMT by upregulating the expression of cysteine-rich angiogenic inducer 61 (Cyr61). Luciferase assays showed that Cyr61 was a direct transcriptional target of FOXK1. Down regulation of Cyr61 decreased FOXK1-enhanced "CRC cell" migration, invasion, and metastasis. Additionally, FOXK1 expression was positively correlated with Cyr61 expression and was associated with poor prognosis. Conclusions: The Snail/FOXK1/Cyr61 signaling axis regulates the EMT and metastasis of CRC.

(C) 2018 The Author(s)

Published by S. Karger AG, Basel
\end{abstract}

Jide Wang

and Side Liu 


\section{Cellular Physiology Cell Physiol Biochem 2018;47:590-603 \begin{tabular}{l|l} 
DOI: 10.1159/000490015 & O 2018 The Author(s). Published by S. Karger AG, Basel \\
www.karger.com/cpb
\end{tabular} \\ Huang et al: Molecular Regulation of CRC Metastasis}

\section{Introduction}

The epithelial-mesenchymal transition (EMT) is a well-recognized process by which cells in a differentiated epithelial state convert into a dedifferentiated mesenchymal state, and plays an essential role in the regulation of embryogenesis, organ fibrosis, wound healing, and cancer metastasis [1-3]. Crucial events of the EMT include decreased expression of the cell-cell adhesion molecule E-cadherin, increased expression of mesenchymal proteins such as vimentin and $\mathrm{N}$-cadherin, and deregulation of the canonical Wnt/ $\beta$-catenin signaling pathway [4-5]. It has been shown that colorectal cancer (CRC) cells that undergo the EMT acquire invasive and migratory properties [5-6]. A number of transcription factors such as Twist [6], Snail [7], Slug, [8] and zinc finger E-box-binding homeobox 1 (ZEB1)/2 [9] are major inducers of the EMT and directly or indirectly repress E-cadherin in CRC cells [10]. Thus, CRC patients may benefit from targeted therapies that inhibit the EMT.

Forkhead box k1 (FOXK1), a transcription factor that belongs to the forkhead family, consists of the winged-helix DNA-binding domain and N-terminal and C-terminal transcriptional domains [11]. FOXK1 has been shown to induce the EMT [12,13] and promote CRC cell invasion in vitro [14]. In addition, we recently found that FOXK1 promotes tumor metastasis through induction of the EMT, thereby maintaining the invasive potential of gastric cancer cells $[13,15]$. Snail is a zinc-finger transcription factor that triggers the EMT by directly repressing E-cadherin expression [7]. Snail overexpression in different epithelial cells leads to conversion from a well-differentiated phenotype to a mesenchymal/ fibroblastic phenotype at the same time that E-cadherin expression is repressed, resulting in the acquisition of invasive and migratory properties [16-17]. Snail binds to the E-box nucleotide sequence CANNTG in the promoter regions of target genes [18]. However, the effects of FOXK1 and Snail on the EMT process in CRC have not been investigated.

Cysteine-rich angiogenic inducer 61 (Cyr61) is a member of the Cyr61/CTGF/Nov (CCN) protein family, which consists of Cyr61 (CCN1), connective tissue growth factor (CTGF/CCN2), and nephroblastoma-overexpressed gene (Nov/CCN3) [19-21]. Cyr61 is up regulated in many cancers, such as breast [19], esophagus [22], and colorectal cancers [23]. Several studies have reported that Cyr61 promotes the EMT and tumor metastasis [24, 25].

In this study, we demonstrated that FOXK1, a direct transcriptional target of Snail, promoted CRC invasion and metastasis by up regulating Cyr61expression. In addition, the Snail/FOXK1/Cyr61 signaling axis was associated with CRC metastasis and indicative of poor prognosis.

\section{Materials and Methods}

\section{Chemicals, reagents, and cell lines}

Mouse E-cadherin (H-108), rabbit anti-FOXK1 (H-140), anti-Cyr61(H-78), mouse anti-glyceraldehydes 3-phosphate dehydrogenase (GAPDH), E-cadherin (H-108), and horseradish peroxidase conjugated secondary antibody were obtained from Santa Cruz Biotechnology (Santa Cruz, CA, USA). Rabbit antivalentine (Ag0489), matrix metalloproteinase-2 (MMP-2) (Ag0549), and MMP-9 (Ag0552) were acquired from Proteintech (Wuhan, China). Snail (095863) was obtained from Abcam (Cambridge, UK). We used two colon carcinoma cell lines: LoVo and SW480, which were purchased from American Type Culture Collection (Rockville, MD, USA) and cultured in RPMI-1640 medium as previously described [12].

\section{Plasmid construction and small interfering RNA transfection}

FOXK1 plasmid was described as in our previous publication [12]. pENTER-Snail and empty vector (pENTER) plasmids encoding a FLAG tag were purchased from Vigene Biosciences (Rockville, MD, USA). Viable clones were pooled, screened for FOXK1 or Snail expression, and maintained in medium containing $600 \mu \mathrm{g} / \mathrm{mL}$ Geneticin Selective Antibiotic (G418;Calbiochem, Darmstadt, Germany) for additional studies. For RNA interference studies, CRC cells were transfected with predesigned small interfering RNAs (siRNAs) at final concentrations of $25 \mathrm{nM}$, as previously described [12, 18]. The siRNA sequences were 
Huang et al: Molecular Regulation of CRC Metastasis

as follows: FOXK1 sense strand: 586-CCAUCAAGAUCCAGUUCAC (dTdT)-605 and Cyr61 sense strand: 876-GCAUCCUAUACAACCCTTTAC-897; scrambled (src) siRNA 5'-UUCUCCGAACGUGUCACGU-3', which did not target any gene, was used as the negative control. The siRNA transfections were performed using Lipofectamine 2000 (Invitrogen, Carlsbad, CA, USA) according to the manufacturer's instructions. At $48 \mathrm{~h}$ post transfection, cells were lysed and western blot analyses were performed. Cells seeded in tissue culture plates were grown to 30-50\% confluence and transfected using Lipofectamine 2000 or LipofectamineRNAiMAX according to the manufacturer's instructions. The effect of gene knockdown was evaluated by western blotting $36 \mathrm{~h}$ post transfection.

\section{Construction of tissue microarrays and immunohistochemistry}

CRC samples and the corresponding adjacent colorectal tissues were used to construct a tissue microarray (TMA; Shanghai Biochip Co., Ltd., Shanghai, China). The tissue microarray was stained for FOXK1, Cyr61, and Snail expression. The TMA was scored independently by two pathologists for both staining intensity and extent of protein expression across the section. Immunohistochemical staining was performed using the Dako Envision Plus System (Dako, Carpinteria, CA, USA) according to the manufacturer's instructions. Analysis was performed by two independent observers who were blinded to the clinical outcome. Staining intensity was scored as 0 , negative staining; 1 , weak staining; 2 , moderate staining; and 3 , intense staining. Cells with negative or weak staining were defined as low expressers, and cells with moderate or intense staining were defined as high expressers, as previously described [12, 15]. This study was approved by the institutional human ethics committee of the relevant institutions.

\section{RNA extraction and quantitative real-time reverse transcription polymerase chain reaction}

Total RNA extraction and reverse transcription were performed as previously described [18]. Quantitative real-time reverse transcription PCR (qRT-PCR) was performed using the Applied Biosystems Sequence Detection System 7900 (ABI Prism 7900HT; Applied Biosystems Inc., Foster City, CA, USA) with a $10 \mathrm{~mL}$ mixture comprising the Power SYBR GREEN PCR Master Mix (Applied Biosystems), $500 \mathrm{nmol}$ of each primer, and $300 \mathrm{ng}$ complementary DNA template. The reactions were performed with an initial denaturation step of $95^{\circ} \mathrm{C}$ for $5 \mathrm{~min}$, followed by 60 cycles of $20 \mathrm{~s}$ at $94^{\circ} \mathrm{C}, 20 \mathrm{~s}$ at $55^{\circ} \mathrm{C}$, and $40 \mathrm{~s}$ at $72^{\circ} \mathrm{C}$. A final extension at $72^{\circ} \mathrm{C}$ for $5 \mathrm{~min}$ was included before a temperature increase from $72^{\circ} \mathrm{C}$ to $95^{\circ} \mathrm{C}$ at $0.1^{\circ} \mathrm{C} / \mathrm{s}$ with continuous fluorescent acquisition. Each complementary DNA sample was run in duplicate for each real-time PCR assay, and average relative fold change in mRNA levels was determined using the 2- $\Delta \Delta \mathrm{Ct}$ method, with GAPDH used as the internal control.

\section{Preparation of cell lysates and immunoblotting}

Culture medium was removed and the cells were washed with cold phosphate-buffered saline (PBS) and lysed in precooled mammalian cell lysis buffer (40 mM HEPES [pH 7.4], 1\% Triton X-100,100mM NaCl, 1mM ethylenediaminetetraacetic acid (EDTA), $25 \mathrm{mM} \beta$-glycerol phosphate, $1 \mathrm{mM}$ sodium orthovanadate, $10 \mu \mathrm{g} /$ $\mathrm{mL}$ leupeptin, and $10 \mu \mathrm{g} / \mathrm{mL}$ aprotinin). The sodium dodecyl sulfate polyacrylamide gel electrophoresis (SDS-PAGE) samples were prepared by directly adding $5 \times$ sodium dodecyl sulfate (SDS) sample buffer to the lysates, followed by vortexing and denaturing at $100^{\circ} \mathrm{C}$ for $5 \mathrm{~min}$. Proteins were resolved on $12 \%$ SDSPAGE gels and electro transferred onto immobilon polyvinylidene fluoride membranes (Millipore, Stafford, VA, USA).The membranes were incubated with primary antibodies overnight at $4^{\circ} \mathrm{C}$, followed by incubation with secondary antibodies for $2 \mathrm{~h}$ at room temperature. The protein bands were detected using the Western Lightning ECL Detection Kit (Beyotime, Beijing, China).

\section{Invasion and cell migration assays}

Invasion and cell migration assays were performed as previously described [26]. Briefly, cells were plated in 6 well plates; once they were $100 \%$ confluent, they were wounded with a pipette tip at time 0 . Media were changed to remove the cell debris and the cells were cultured in the presence of $10 \mu \mathrm{g} / \mathrm{mL}$ mitomycin $\mathrm{C}$ to inhibit cell proliferation. Photographs were taken after $60 \mathrm{~h}$. Cells were plated in serumfree medium on transwell inserts (Corning, NY, USA) coated with $25 \mu$ g Matrigel (BD Biosciences, San Jose, CA, USA) for the invasion assays. After incubation for $48 \mathrm{~h}$ at $37^{\circ} \mathrm{C} / 5 \% \mathrm{CO} 2$, the inserts were fixed in $3.7 \%$ paraformaldehyde/PBS and stained with $2 \%$ crystal violet. The number of invading/migrating cells was counted in five representative fields $(200 \times)$ per insert. 


\section{Cellular Physiology Cell Physiol Biochem 2018;47:590-603 \begin{tabular}{l|l} 
and Biochemistry Published on/Ine:1V0015 25, 2018 & $\begin{array}{l}\text { (c) 2018 The Author(s). Published by S. Karger AG, Basel } \\
\text { www.karger.com/cpb }\end{array}$ \\
\hline
\end{tabular}}

Huang et al: Molecular Regulation of CRC Metastasis

Promoter reporter and dual luciferase assays

First, 482 base pairs (bp) (FOXK1p1), 597bp (FOXK1p2), and 31bp (Cyr61p) fragments of the FOXK1 and Cyr61 promoter upstream of the transcription start site were cloned into the pGL3 basic vector. For the luciferase assay, cells were transiently transfected with the various pLuc constructs using Lipofectamine 2000. The firefly and Renilla luciferase activities were measured using the Dual-Luciferase reporter system (Promega, Madison, WI, USA) with a luminometer (TD-20/20; EG\&G Berthold, Australia). The firefly luciferase enzyme activity was normalized to the renilla luciferase enzyme activity. The transcriptional activity at the promoter was presented as the fold induction of relative luciferase units (RLUs) compared with the basic pGL3 vector control. The RLU was the value of the firefly luciferase unit divided by the value of the Renilla luciferase unit. All treatments were performed in triplicate for each experiment. Site-directed mutagenesis of potential Snail or Cyr61 binding sites was conducted in the FOXK1p1, FOXK1p2, and Cyr61 plasmids using the Clon Express II One Step Cloning Kit (Vazyme, Nanjing, China). All mutations were verified by sequencing.

\section{Chromatin immunoprecipitation assay}

Cells that were transfected with the appropriate plasmids were cross-linked in $1 \%$ formaldehyde at $37^{\circ} \mathrm{C}$ for $10 \mathrm{~min}$. After washing with PBS, cells were resuspended in $300 \mathrm{~mL}$ lysis buffer (50 mM Tris [pH 8.1], $10 \mathrm{mM}$ EDTA, 1\%SDS, and $1 \mathrm{mM}$ phenylmethylsulfonyl fluoride). DNA was sheared to small fragments by sonication. Supernatants were precleared using herring sperm DNA/Protein G-Sepharose slurry (SigmaAldrich, St. Louis, MO, USA). Recovered supernatants were incubated with specific antibodies or an isotype control immunoglobulin $\mathrm{G}$ for $2 \mathrm{~h}$ in the presence of herring sperm DNA and protein G-Sepharose beads. Immunoprecipitated DNA was retrieved from the beads with $1 \%$ SDS and a 1. 1M NaHCO3 solution at $65^{\circ} \mathrm{C}$ for $6 \mathrm{~h}$. Then the DNA was purified using a PCR Purification Kit (Qiagen, Valencia, CA, USA).

\section{Immunofluorescence}

For staining of F-actin, cells were washed with PBS and fixed in methanol/acetone (1:1) for 5 min on ice, followed by incubation with rhodamine-conjugated phallotoxin ( $5 \mathrm{U} / \mathrm{mL}$, Molecular Probes, Eugene, OR, USA) in PBS at a 1:40 dilution for 1h. Coverslips were washed, stained with DAPI, and mounted on slides. Nuclei were stained with $1 \mu \mathrm{g} / \mathrm{mL}$ Hoechst 33258, and cells were visualized by fluorescence microscopy.

Construction of lentivirus and stable cell lines

Lentiviral vectors encoding short hairpin RNAs (shRNAs) were generated using PLKO.1-TRC (Addgene, Cambridge, MA, USA) and designated as LV-shFOXK1, LV-shCyr61, and LV-shcontrol (non-target shRNA control). The vector "pLKO.1-puro Non-Target shRNA Control Plasmid DNA" (SHC016;Sigma) contained an shRNA insert that did not target any known genes from any species. The shRNA sequences were: shFOXK1, 5'-CCAUCAAGAUCCAGUUCAC-3; and shCyr61, 5'-GCATCCTATACAACCCTTTAC-3. Lentiviral vectors encoding human FOXK1 and Snail genes were constructed in FUW-teto (Addgene) and designated as LV-FOXK1 and LVSnail, respectively. An empty vector, designated LV-control, was used as the negative control. Concentrated lentivirus was transfected into CRC cells at a multiplicity of infection ranging from 30 to 50 in the presence of polybrene ( $6 \mu \mathrm{g} / \mathrm{mL}$ ).After $72 \mathrm{~h}$, CRC cells were selected for 2 weeks using $2.5 \mu \mathrm{g} / \mathrm{mL}$ puromycin (OriGene Technologies Inc., Rockville, MD, USA). Selected pools of knockdown and over expressing cells were used for subsequent experiments.

\section{In vivo metastatic model and bioluminescent imaging}

BALB/C nude mice (4-6 weeks old) were housed under standard conditions and cared for according to the institutional guidelines for animal care. All animal experiments were approved by the Committee on the Use of Live Animals in Teaching and Research, Southern Medical University (Guangdong, China). The 4-6-week-old BALB/C-nu/nu nude mice were obtained from the Laboratory Animal Unit, Southern Medical University. To evaluate the metastatic potential of CRC cells in vivo,SW480/pEGFP-N1, SW480/ pEGFP- FOXK1, SW480/pEGFP-src shRNA, and SW480/pEGFP-FOXK1 shRNA-transfected cells (density of $5 \times 106$ cells/plate)were inoculated into the dorsal subcostal incision to expose the spleen $(\mathrm{n}=3$ for each group). A $50 \mu \mathrm{L}$ volume of tumor cell suspension was slowly injected into the spleen using a 25-gauge needle. After 42 days, the mice were sacrificed, and individual organs were removed and assessed using the In-Vivo Imaging System (Kodak, Rochester, NY, USA). The metastatic tissues were analyzed by hematoxylin and eosin staining, immunohistochemistry (IHC), and qRT-PCR. 


\section{Cellular Physiology Cell Physiol Biochem 2018;47:590-603

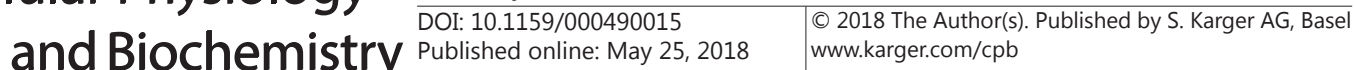 \\ Huang et al: Molecular Regulation of CRC Metastasis}

\section{Statistical analysis}

Quantitative data obtained from experiments with biological replicates are shown as the mean \pm standard deviation. Comparisons between groups were conducted using the Student's T-test. Linear regression and Pearson correlation analysis were performed in the TMAs. Survival analysis was performed with the Kaplan-Meier and Log-rank tests. The Cox proportional hazards model was used to determine the independent factors that influenced survival and recurrence based on variables selected from the univariate analysis. P-values less than 0.05 were considered statistically significant.

\section{Results}

Snail promoted CRC tumorigenesis and invasion by up regulating FOXK1 expression

It was previously revealed that overexpression of Snail up regulated FOXK1 in SW480 CRC cells using gene expression profiling (https://www.ncbi.nlm.nih.gov/ geoprofiles/100447371). Here, we first investigated whether FOXK1 and Snail expression levels were correlated in CRC. To this end, we analyzed the expression of FOXK1 and Snail in eight freshly collected CRC biopsies. Western blot analyses indicated that both FOXK1 and Snail were significantly up regulated in all tumor samples examined, compared with the paired adjacent noncancerous tissues from the same patients (Fig. 1A). The protein expression of FOXK1 was positively correlated with Snail expression (Fig. 1B). In addition, Spearman correlation analysis showed a positive correlation between FOXK1 and Snail expression (Fig. 1C). Moreover, the downregulation of Snail decreased FOXK1 expression in three colon cancer cell lines (Fig. 1D). Next, we analyzed FOXK1 and Snail expression patterns using TMAs. We found that positive FOXK1 and Snail signals were strongly expressed in only the carcinoma cells of CRC samples as shown in Fig. 1E. In contrast, adjacent normal

Fig. 1. Snail transactivates FOXK1 expression in CRC cells. (A)(C) Western blot analysis of FOXK1 and Snail protein expression in eight freshly collected CRC biopsies (A). Relative protein expression levels were quantified by comparing the gray level of each band using Quantity One Software (B). Spearman's correlation analysis was used to determine the relationship between FOXK1 and Snail protein expression (C). (D) Western blot analysis of FOXK1 and Snail expression in the indicated CRC cells. (E) FOXK1 and Snail protein expression in CRC tissues as detected by TMA. (F) The luciferase (Luc) reporter

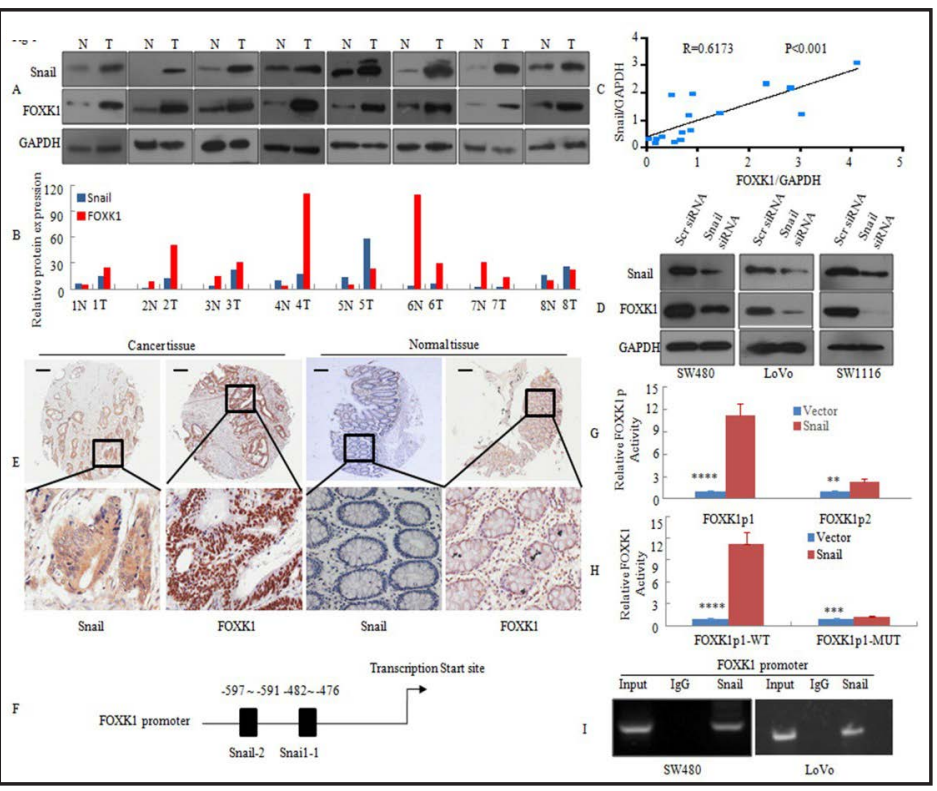
constructs contained the FOXK1 promoter with two potential Snail binding sites upstream of the luciferase gene.(G) Snail activated the FOXK1 promoter (FOXK1p) after co-transfection in SW480 cells. Luciferase activity was measured $48 \mathrm{~h}$ after transfection. Luciferase activity is expressed as the ratio of promoter reporter activity to control vector luciferase activity. RLU, relative luciferase unit; ${ }^{* *} \mathrm{P}<0.05$, ${ }^{* * * *} \mathrm{P}<0.001$. (H)Site-directed mutagenesis of FOXK1p1-WT (wild type) was performed to generate FOXK1p1-MUT. ${ }^{* * * *} \mathrm{P}<0.001,{ }^{* * *} \mathrm{P}<0.01$. (I) ChIP assay was performed using an anti-Snail antibody or control IgG. The FOXK1 promoter region to which Snail bound was significantly enriched after immunoprecipitation with an anti-Snail antibody. PCR products following ChIP were run on an ethidium-stained gel. Scale bars: $200 \mu \mathrm{m}$ in E.

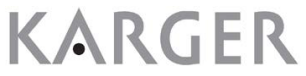




\section{Cellular Physiology \begin{tabular}{l|l} 
and Biochemistry Published 10.1159/000490015 & $\begin{array}{l}\text { C) } 2018 \text { The Author(s). Published by S. Karger AG, Basel } \\
\text { www.karger.com/cpb }\end{array}$ \\
\hline
\end{tabular} \\ Huang et al: Molecular Regulation of CRC Metastasis}

colon tissues did not express FOXK1 or Snail protein. Protein-protein interaction analysis using the STRING database showed that there was no interaction between the two proteins (https://string-db.org/).To determine whether Snail could bind to the FOXK1 promoter, we scanned the promoter region $(<600 \mathrm{bp})$ of human FOXK1 for the E-box consensus sequence and found two potential binding sites: -476 to -482 (E-box 1, Snail1) and -576 to -582 (E-box 1, Snail2) from the transcription initiation site (Fig. 1F).We cloned the promoter regions E-box 1 (FOXK1p1) and E-box 2 (FOXK1p2) of human FOXK1 upstream of a luciferase gene in a reporter plasmid. Transient transfections were performed to investigate whether the FOXK1 promoter was activated by Snail overexpression. Dual-luciferase assays showed that FOXK1p1 activity in Snail cells increased 11-fold compared with empty vectortransfected cells, whereas the magnification exhibited a slight decrease with FOXK1p2 transfection (Fig. 1G). To verify the functional relationship between E-box binding site 1 and FOXK1 promoter activity, a mutation was introduced into FOXK1p1 (FOXK1p1-mut1) by site-directed mutagenesis that eliminated its transcriptional activity (Fig. $1 \mathrm{H}$ ).To confirm that Snail could physically bind to the FOXK1 promoter in vivo, we performed chromatin immunoprecipitation (ChIP) assays in CRC cell lines expressing endogenous Snail. The FOXK1 promoter region containing site 1 (E-box 1) exhibited significant enrichment after immunoprecipitation with an anti-Snail antibody (Fig. 1I).These studies suggested that Snail promoted CRC tumorigenesis and invasion by transactivating FOXK1 expression.

Fig. 2. Snail-FOXK1 axis promotes EMT-mediated CRC invasion and metastasis. (A) Expression levels of Snail were detected by western blot analysis in SW480 cells, which were transfected with Snail-overexpressing plasmids, followed by transfection with FOXK1 siRNA or scr siRNA as a negative control. (B) Morphology of stable cells expressing Snail transfected with FOXK1 siRNA or src siRNA in CRC cells, as analyzed by phase-contrast microscopy. (C) SW480 cells stained with rhodamine-phallotoxin for $48 \mathrm{~h}$ to identify F-actin filaments were visualized under a fluorescent microscope. (D) EMT biomarkers including E-cadherin, vimentin, MMP2, MMP9, FOXK1, and Snail were detected by western blotting $48 \mathrm{~h}$ after transfection. (E) Vector, stable Snail transfectants were transfected with FOXK1 siRNA after $48 \mathrm{~h}$, and the invasive ability of the cells decreased. Invasive

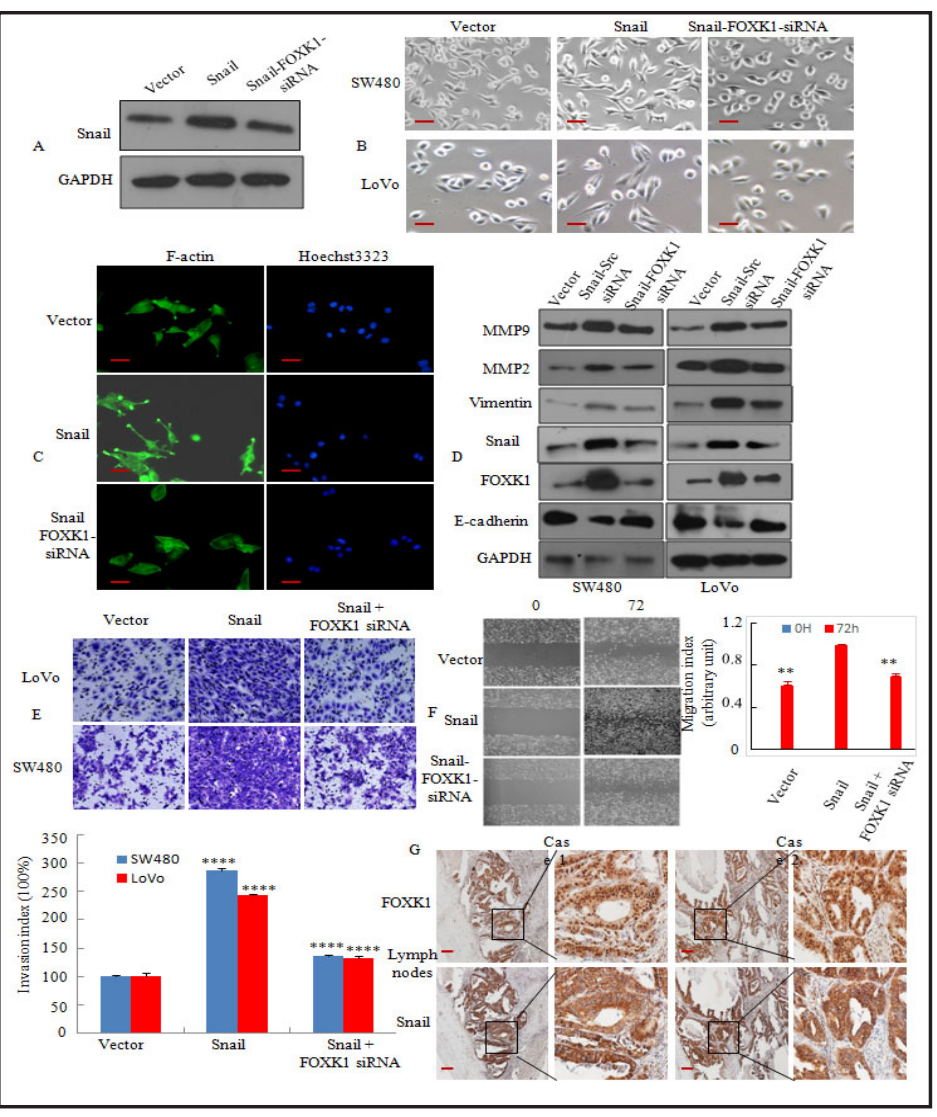
potential of vector, Snail, and Snail-FOXK1 siRNA. ${ }^{* * *} \mathrm{P}<0.001$.(F) For the woundhealing experiments, cells were analyzed with live-cell microscopy. Original magnification, $10 \times$. SW480 cells were analyzed with live-cell microscopy. ${ }^{* *} \mathrm{P}<0.05$. (G) Representative IHC images are shown for Snail and FOXK1 expression in lymph node metastatic cancer tissues. Scale bars: $20 \mu \mathrm{m}$ in B, $50 \mathrm{~mm}$ in C, $100 \mu \mathrm{m}$ in G. Pictures are representative of three independent experiments with identical results. 
Snail-FOXK1 axis promotes EMT-mediated CRC cells invasion and metastasis

To investigate whether Snail regulates FOXK1 in CRC, we downregulated FOXK1 in Snailoverexpressing SW480 cells using siRNA, and confirmed FOXK1 knockdown by western blot analysis (Fig. 2A). Then we examined the morphologic features of these cells. Pooled stable vector transfectants displayed a round or flat morphology with short cytoplasmic processes. However, Snail transfectants exhibited a spindle-like, fibroblastic morphology, which is one of the main EMT characteristics. Conversely, FOXK1 knockdown in Snail-overexpressing cells led to EMT reversion (Fig. 2B).Some reports have shown actin cytoskeleton regulation of the EMT in metastatic cancer cells $[12,26]$. Thus, we stained F-actin with phalloidin, followed by immunofluorescence analysis, which showed that Snail-overexpressing cells had an elongated cellular morphology and the presence of F-actin fibers compared with vector cells, a hallmark of the mesenchymal phenotype. Conversely, Snail-overexpressing cells with FOXK1 knockdown exhibited a cobblestone morphology and small F-actin fibers, indicative of a reverse in the EMT process (Fig. 2C).Moreover, expression of a typical EMT epithelial marker, E-cadherin, was unregulated after FOXK1 knockdown in Snail-overexpressing cells. In contrast, the mesenchymal markers MMP-2, MMP-9, and vimentin were downregulated (Fig. 2D). In addition, knockdown of FOXK1 in Snail-overexpressing cells led to a decrease in the migratory potential of Snail-overexpressing cells in vitro, as determined by the wound healing assay (Fig. 2E). Similarly, FOXK1 downregulation in Snail-overexpressing cells decreased the invasion potential of Snail-overexpressing cells (Fig. 2F). To validate our findings in vivo, we evaluated Snail and FOXK1 expression in serial sections of lymph node metastatic cancer tissues from two patients and found that Snail and FOXK1 were expressed at high and intermediate levels, respectively (Fig. 2G). Taken together, these data suggest that the Snail-FOXK1 axis promotes EMT-mediated CRC cell invasion and metastasis.

Dysregulation of the Snail-FOXK1 axis is associated with poor prognosis and aggressive progression of CRC

To explore the clinical relevance of FOXK1 and Snail expression, we analyzed their clinicopathological features in CRC. In tumor samples obtained from 100 patients, FOXK1 and Snail expression was significantly correlated with tumor differentiation, lymph node metastasis, and serosal invasion; however, the expression levels were not correlated with age, gender, American Joint Committee on Cancer stage, location, or tumor size. Next, we investigated the correlation between FOXK1 and Snail expression in 100 pairs of adjacent normal colon mucosal tissues and cancer tissues. Semi-quantitative scoring showed that both proteins were expressed at higher levels in cancer tissues than in adjacent normal colorectal mucosa tissues (Fig. 3A). After calculating the regression coefficient between the expression scores of FOXK1 and Snail, we observed a significant correlation between FOXK1 and Snail $(\mathrm{R}=0.6064)$ in primary CRC (Fig. 3B).To analyze the correlation between Snail or FOXK1 expression and the prognosis of CRC patients, Kaplan-Meier survival curves were generated. A high positive expression of each protein was correlated with poor outcome (Fig. 3C (a) and (b)).Double-positive cases that expressed both proteins showed the worst prognosis (Fig. 3C (c)). Representative IHC images for the tissues are shown in Fig. 3D. To examine the role of the Snail-FOXK1axis in the progression of CRC, we injected enhanced green fluorescent protein (EGFP)-N1, EGFP-Snail, and EGFP-Snail-FOXK1 shRNA-transfected cells expressing green fluorescent protein into nude mice to examine the liver metastasis (Fig. 3E). At 42 days after injection, mice injected with Snail-overexpressing SW480 cells but not vector-expressing SW480 cells had large metastatic nodules in their livers, whereas mice with FOXK1 knockdown Snail-overexpressing cells had small liver nodules compared with those in the scr shRNA-transfected Snail-overexpressing cells (Fig. 3F). The presence of liver metastases from CRC was confirmed by histological analysis (Fig. 3G). As further confirmation that the Snail-FOXK1axis correlates with the EMT, FOXK1 expression was repressed in Snail-overexpressing cells in orthotopic xenograft tumors. Snail overexpression resulted in significant downregulation of E-cadherin in an IHC assay (Fig. $3 \mathrm{H}$ ), whereas loss of FOXK1 in Snail-overexpressed cells caused an increase in E-cadherin expression, as 
Fig. 3. Dysregulated Snail-FOXK1 axis is associated with poor prognosis and aggressiveness of CRC. (A) Average scores of the two proteins in normal and CRC tissues. (B) Positive staining for Snail and FOXK1 was quantified, and their correlation was analyzed using the Spearman's correlation method. (C) Kaplan-Meier overall survival analysis of CRC patients. Survival analysis was performed according to the expression status of Snail (a), FOXK1 (b), and the combined expression of Snail and FOXK1 (c). (D) Representative IHC images of the tissues are shown. (E) Mice orthotopically transplanted showing external whole-body fluorescence images of SW480 cells $(\mathrm{n}=3$ in each group). (F)Metastatic cancer tissues were stained with H\&E. (G) \& $(\mathrm{H})$ Expression of E-cadherin in tumors derived from SW480 cells was determined using IHC staining and qPCR. $* * * * \mathrm{P}<0.001$, vector vs. Snail, Snail vs. Snail-FOXK1 shRNA.

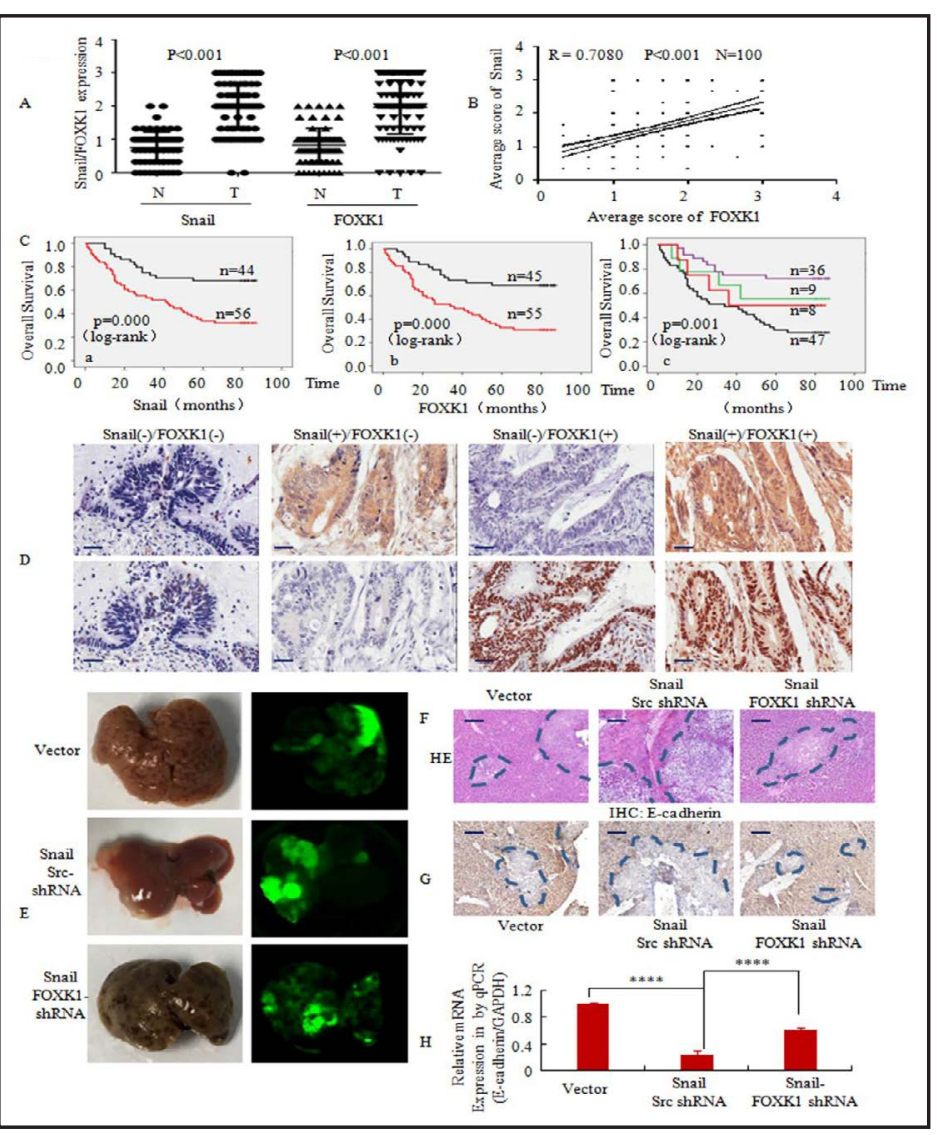
Scale bars: $100 \mu \mathrm{m}$ in D, Fand G.

determined by qPCR (Fig. 3I). Taken together, these results indicate that the Snail-FOXK1 axis plays an important role in the EMT and metastasis in CRC.

\section{Cyr61 is a direct target for transcriptional activation by FOXK1}

To examine the role of FOXK1 as a transcription factor and to explore its target genes, we performed a TMA using SW480 CRC cells transfected with FOXK1-targeting siRNA or control siRNA. A total of 491 genes were upregulated, and 398 were downregulated. Cyr61 was the most significantly downregulated gene. Because Cyr61 is a key regulator of migration/invasion and the EMT in CRC cells [27, 28], we focused on Cyr61 as a target of FOXK1. To confirm the TMA data, Cyr61 downregulation by FOXK1siRNA was examined by western blot analysis in SW480 cells. Transfection of FOXK1 siRNA downregulated Cyr61 protein expression (Fig. 4A). In addition, downregulation of Cyr61 by transfection of FOXK1siRNA in other cell lines (LoVo and SW1116) yielded similar results (Fig. 4A). Then, we investigated whether Cyr61 was directly regulated by FOXK1. Yang et al [29]. showed that FOXK recognizes the DNA sequence motif, WRTAAAAYA, and regulates the p21, c-Myc, and cyclin-dependent protein kinase 2 genes. We evaluated Cyr61 in the proximal promoter $(<600 \mathrm{bp}$ ) regions of FOXK1 and identified one potential FOXK1-binding site at -25 to -31 (Fig. 4B). The luciferase reporter assay showed that FOXK1 transactivated Cyr61 promoter (Cyr61p) activity. Moreover, site-directed mutagenesis showed that FOXK1-binding sites were critical for FOXK1-induced Cyr61 transactivation (Fig. 4C). A ChIP assay confirmed the direct binding of FOXK1 to the Cyr61p promoter in CRC cells (Fig. 4D). These studies suggest that FOXK1 binds to the Cyr61 promoter and upregulates Cyr61 transcriptional activity. Then, we used western blotting to examine FOXK1 and Cyr61 expression in eight pairs of human CRC tissues and matched non-cancerous colon mucosa. As shown in Fig. 


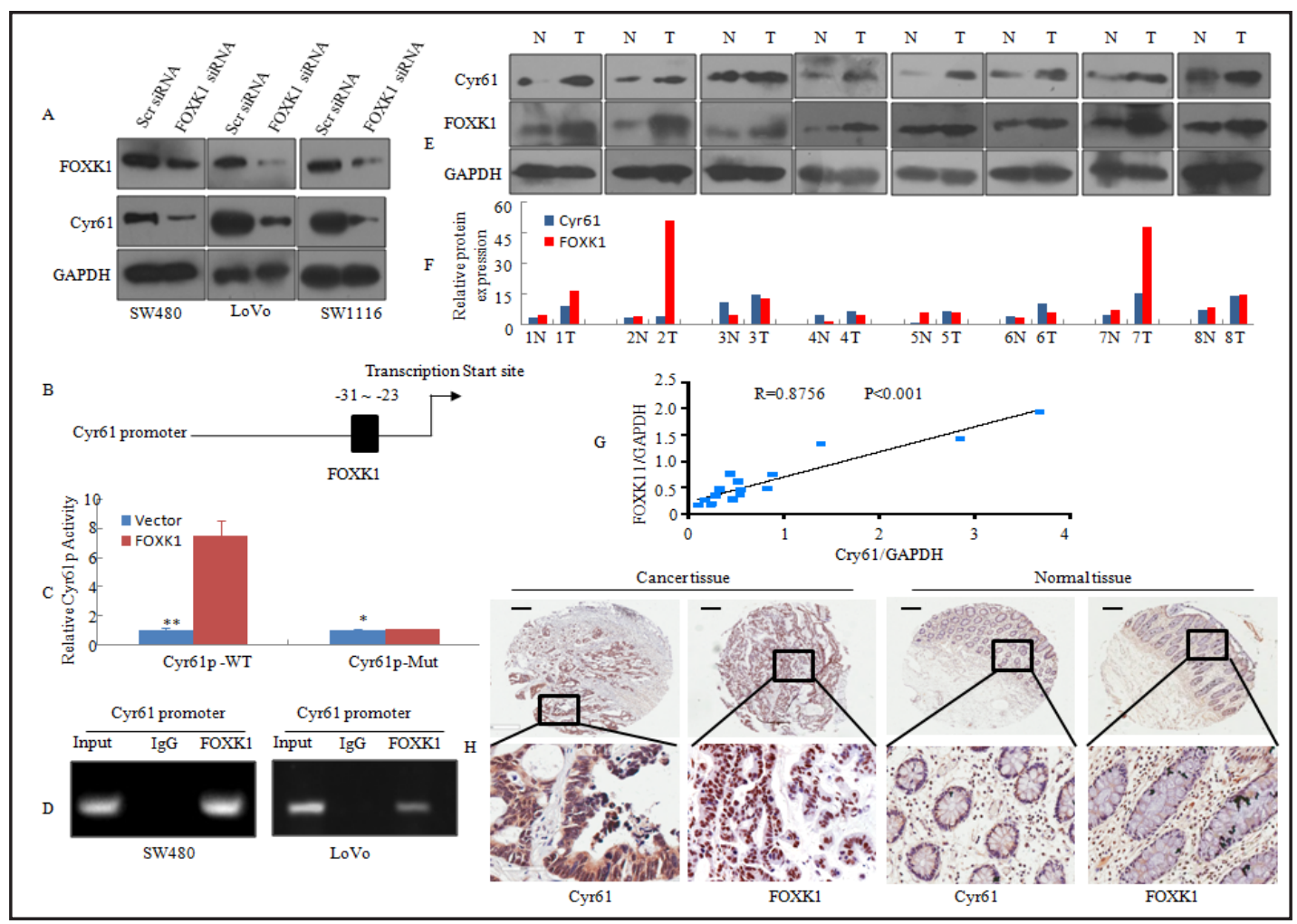

Fig. 4. Cyr61 is a direct target for transcriptional activation by FOXK1. (A)Western blot analysis of FOXK1 and Cyr61 expression in the indicated CRC cells.(B) Schematic representation of the promoter region of Cyr61 (Cyr61p). (C)FOXK1 transactivates the Cyr61 promoter. Selective mutation analysis identified oneFOXK1responsive site in the Cyr61 promoter. Luciferase activity was measured $48 \mathrm{~h}$ after transfection. ${ }^{* * * *} \mathrm{P}<0.001$. (D)ChIP was performed using an anti-FOXK1 antibody or control IgG. (E)Western blot analysis of FOXK1 and Cyr61 protein expression in eight freshly collected CRC biopsies. (F)Relative protein expression levels were quantified by comparing the gray level of each band using Quantity One Software. (G)Spearman's correlation analysis was used to determine the relationship between FOXK1 and Cyr61 protein expression. (H)FOXK1 and Snail protein expression in CRC tissues was detected by TMA. Scale bars: $200 \mu \mathrm{m}$ in $\mathrm{H}$.

4E, FOXK1 and Cyr61 were significantly upregulated in the eight tumor samples examined compared with the paired adjacent noncancerous tissues from the same patients. The protein expression of FOXK1 was positively correlated with Cyr61 protein expression (Fig. 4F). In addition, a positive correlation between FOXK1 and Cyr61 expression was found using spearman rank correlation analysis (Fig. 4G). Next, we investigated the correlation between Cyr61 and FOXK1 expression using TMAs of adjacent normal colon mucosal tissues and cancer tissues. Both proteins were highly expressed in the cancer tissues, but they were either not expressed or were expressed at low levels in normal tissues (Fig. 4H). These data indicate that FOXK1 transactivates Cyr61 expression and both proteins are associated with tumorigenesis and metastatic potential of CRC.

FOXK1 is critical for Cyr61-mediated EMT phenotypes and metastatic potential in vitro

To investigate whether FOXK1 regulates Cyr61 in CRC, we used siRNA to downregulate Cyr61 expression in FOXK1-overexpressing cells, which was confirmed by western blotting (Fig. 5A). Then, we examined the morphologic features of the transfected cells. FOXK1 transfectants exhibited long or dendritic-like cytoplasmic processes that were visible by a phase-contrast microscope. Cyr61 knockdown in FOXK1-overexpressing CRC cells exhibited a cobblestone morphology, indicative of reverse EMT (Fig. 5B). Then, we stained F-actin using phalloidin. Compared with the vector-expressing cells, FOXK1-overexpressing cells showed 


\section{Cellular Physiology

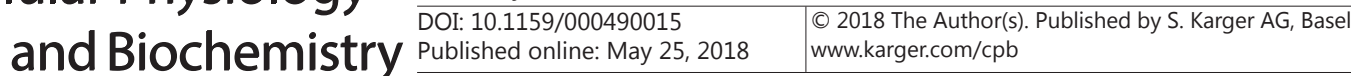 \\ Huang et al: Molecular Regulation of CRC Metastasis}

F-actin staining throughout the cytoplasm and protrusion of the leading edge (Fig. 5C). Conversely, Cyr61 knockdown in FOXK1-overexpressing cells led to weak and aggregated F-actin filaments that were involved in cancer cell invasion and metastasis. SiRNA-mediated repression of Cyr61 was performed in FOXK1 cells and resulted in decreased vimentin expression as well as decreased expression of mesenchymal markers (vimentin, MMP2, and MMP9) and increased expression of the epithelial marker (E-cadherin) compared with control (FOXK1) cells (Fig. 5D).

Next, we performed the wound healing assay. Knockdown of Cyr61 in FOXK1overexpressing cells led to the decreased migratory and invasion potential of FOXK1overexpressing cells in vitro (Fig. 5E,).In addition, we evaluated FOXK1 and Cyr61expression in regional lymph node metastases in CRC tissue and found a positive correlation between FOXK1 and Cyr61expression using IHC (Fig. 5G).Taken together, these data suggest that the co-expression of FOXK1 and Cyr61 regulate cell migration, invasion, and the EMT in CRC cells.

Co-expression of FOXK1 and Cyr61 predicts poor prognosis and promotes invasion/ metastasis of CRC cells

To detect the clinical relevance of FOXK1 and Cyr61 expression, we analyzed their clinicopathological features using TMAs in 100 CRC patients. Similar to FOXK1 and Snail expression patterns, FOXK1 and Cyr61 expression was significantly correlated with tumor differentiation, lymph node metastasis, and serosal invasion. Co-expression of FOXK1 and Cyr61 was associated with CRC progression. To validate our findings in vivo, we investigated FOXK1 and Cyr61 expression in 100 pairs of adjacent normal colon mucosal tissues and cancer tissues. Semi-quantitative scoring of FOXK1 and Cyr61 proteins showed that the

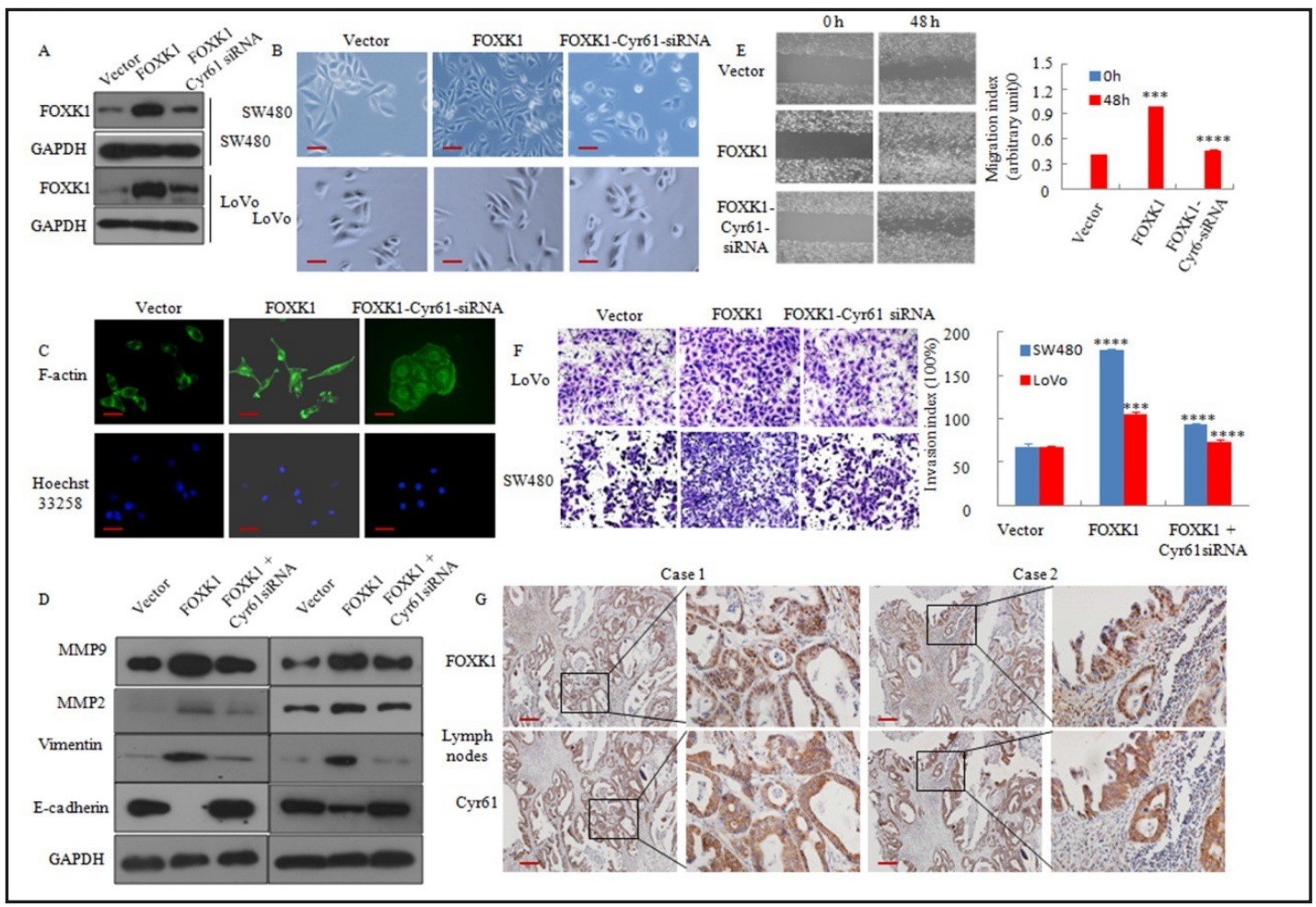

Fig. 5. FOXK1 is critical for Cyr61-mediated EMT phenotypes and metastatic potential in vitro. (A) Expression levels of FOXK1 were detected by western blot analysis in SW480 cells. (B) Morphology of CRC cells was observed byphase-contrast microscopy. (C) SW480 cells stained with rhodamine-phallotoxin by fluorescent microscopy. (D) EMT biomarkers were detected by western blotting. (E) \&(F) Cell migration and invasion assays were performed. (G) Representative IHC images are shown for Cyr61 and FOXK1 expression in lymph node cancer tissues. Scale bars: $20 \mu \mathrm{m}$ in B, $50 \mathrm{~mm}$ in C, $100 \mu \mathrm{m}$ in G.

\section{KARGER}


Fig. 6. Co-expression of FOXK1 and Cyr61 predicts poor prognosis and promotes invasion/metastasis of CRC cells. (A) Average scores of the two proteins in normal and cancerous CRC tissues. (B) Positive staining for Cyr61 and FOXK1 was quantified, and their correlation was analyzed with the Spearman rank-order correlation. (C) KaplanMeier overall survival analysis of CRC patients. (D) Representative IHC images for tissues are shown. (E) Mice orthotopically transplanted showing external whole-body fluorescence images of SW480 cells ( $\mathrm{n}=3$ in each group). (F) Liver tissues were stained with H\&E. (G) \& (H) E-cadherin expression in tumors derived from SW480 cells was determined by IHC staining and qPCR. ${ }^{* * * *} \mathrm{P}<$ 0.001 , vector vs. FOXK1, FOXK1 vs. FOXK1-Cry61 shRNA. Scale bars: $100 \mu \mathrm{m}$ in $\mathrm{D}, \mathrm{F}$ and $\mathrm{G}$.

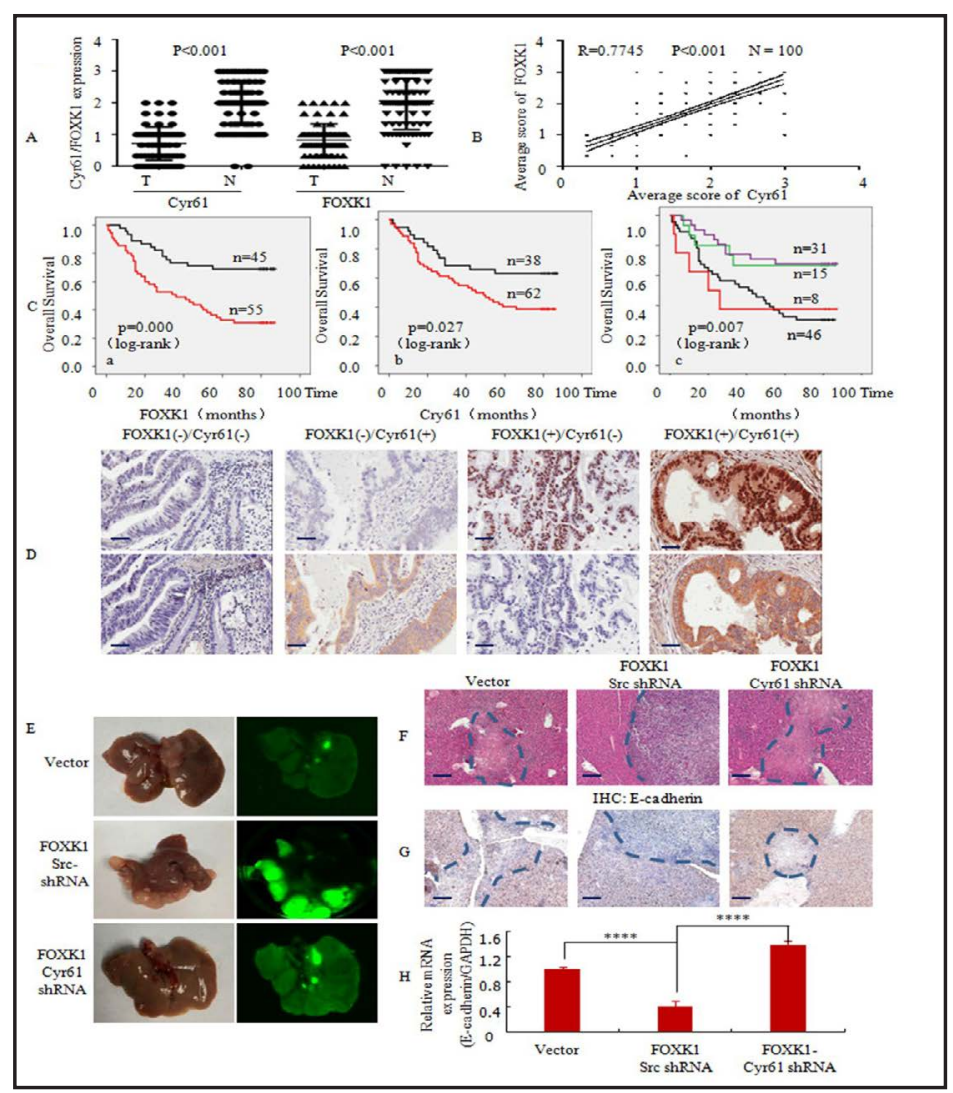

expression of both proteins in cancerous tissues was significantly higher than that of adjacent normal colon tissues (Fig. 6B, P < 0.001), and Spearman's correlation analysis showed a positive correlation between FOXK1 and Cyr61 expression (correlation coefficient $r=0.6748, \mathrm{P}<0.001$, Fig. 6B).Then, we analyzed the correlation between FOXK1 and Cry61 expression and the prognosis of CRC patients. Kaplan-Meier analysis showed that the high positive expression of each protein was correlated with a poor outcome (Fig. 6C (a) and (b)).Double-positive cases that expressed both proteins had the worst prognosis (Fig. 6C (c)). Patients with positive co-expression of FOXK1 and Cry61 had poorer prognosis. Representative IHC images for tissues are shown in Fig. 6D.To investigate whether FOXK1 is critical for Cyr61-mediated EMT regulation invivo, shRNA-mediated repression of Cyr61 was performed in FOXK1-overexpressing SW480 cells after orthotopic implantation assays (Fig. 6E). Metastatic nodules in their livers of the FOXK1-overexpressing group were significantly increased compared with those of the vector group ( $\mathrm{P}<0.001$ vs. vector), whereas liver metastatic nodules after Cyr61 knockdown were greatly decreased compared with those in the FOXK1-overexpressing group (Fig. 6E). The presence of CRC metastasis in the liver was confirmed by histological analysis (Fig. 6F). Moreover, FOXK1 overexpression resulted in significant loss of the epithelial marker E-cadherin, whereas Cyr61 downregulation in FOXK1-overexpressing cells increased E-cadherin expression, as determined by IHC and qRT-PCR (Fig. 6G \& H). Thus, FOXK1 appears to play a critical role in inducing the EMT via Cyr61expression.

\section{Discussion}

The EMT is a key process that drives cancer metastasis, and downregulation of E-cadherin is a major hallmark of this process $[6,12,27]$. The EMT also relies on genomewide transcriptional reprogramming and global chromatin modification [30]. In this study, we found that the FOXK1 promoter activated Snail and FOXK1 directly transactivated Cyr61 gene expression, thereby contributing to the EMT, and the invasion and migration of CRC

\section{KARGER}


cells. In addition, patients with high expression of FOXK1 and Snail or Cyr61 had poorer prognosis than those with low expression. These findings suggest that the Snail-FOXK1Cyr61 axis pathway plays a pivotal role in the EMT and metastasis in CRC.

FOXK1 is a proto-oncoprotein that is overexpressed in various cancer types [31-33]. Ectopic expression of FOXK1 markedly enhances cell proliferation, migration, and invasion in gastrointestinal cancer cells $[15,26]$. We recently demonstrated that FOXK1 induces EMT progression and predicts poor prognosis in human gastric cancer [15, 34]. The Snail super family of zinc-finger transcription factors is involved in processes that cause pronounced cell movements, both during embryonic development and in the acquisition of invasive and migratory properties during tumor progression [16-18]. Snail and Slug [35], Twist1 [6], ZEB1 [3], and ZEB2 [9] transcription factors act as molecular triggers of the EMT in human cancers. However, the molecular mechanisms underlying the correlation between FOXK1 and Snail in cancer progression remain unclear. A recent study implicated Snail in E-cadherin repression via its ability to bind to elements in the E-cadherin promoter [36]. Here, we analyzed the FOXK1 promoter sequence and found two potential transcription factor Snail-binding sites. Luciferase reporter assays, site-directed mutagenesis, and ChIP assays showed that the overexpression of FOXK1 induced by Snail was dependent on Snailbinding sites within the FOXK1 promoter. Thus, Snail upregulated FOXK1 expression and transactivated its promoter activity. Furthermore, Snail expression was positively correlated with FOXK1 expression. These studies suggest that Snail-induced FOXK1 overexpression plays an important role ininducing the EMT and the aggressiveness of CRC.

Cyr61 is a multifunctional protein involved in human CRC development and progression [23]. Overexpression of Cyr61 may induce the EMT, thereby leading to cancer cell invasion, metastasis, and poor prognosis [24, 27]. The Cyr61 promoter contains several definitive DNA regulatory elements such as the p53-binding domain, SMAD binding element, and HMGA1 and YY1 elements [37]. A recent report showed that the Cyr61 promoter contains a potential Sox4 binding site (-168 bp to-162 bp) and transactivates Cyr61 expression [38]. Microarray analysis showed FOXK1 was involved with Cyr61 in CRC cells. Then, we showed that the Cyr61 promoter contains a putative FOXK1 binding site and recognizes the DNA sequence motif WRTAAAAYA (600bp)[29]. Indeed, we showed that FOXK1 binds to a segment of the Cyr61 promoter, indicating that it directly transactivates Cyr61 gene expression.

TMA, western blotting, and immunofluorescence demonstrated that the distribution pattern of FOXK1 was highly congruent with that of Snail or Cyr61, and that FOXK1 protein expression was highly correlated with Snail or Cyr61 expression. Moreover, a linear correlation between FOXK1 and Snail or FOXK1 and Cyr61 expression was observed in CRC. It was previously reported that Snail is expressed in CRC and predicts the clinical outcome of CRC patients [39]. Similar to the results obtained in a previous study [40], we showed that cooperation between FOXK1 and Snail or co-expression of FOXK1 with Cyr61 predicts poor prognosis. Thus, the results of the present study confirm that FOXK1 and/or Snail and/or Cyr61 overexpression may serve as an unfavorable prognostic biomarker for patients with CRC.

\section{Conclusion}

We report that the transcription factor Snail transactivates FOXK1 expression by directly binding to its promoter, which contributes to FOXK1 overexpression in human CRC. Moreover, FOXK1 promotes the EMT and tumor invasion and metastasis by transactivating Cyr61 expression in CRC cells. Thus, targeting the FOXK1-Cyr61-Snail axis may provide a potential therapeutic target for the treatment of CRC.

\section{Acknowledgements}

This study was supported by grants from the National Natural Science Funds of China (Nos.81672875 and 81772964), the President Foundation of Nanfang Hospital, Southern Medical University (Nos.2012B009 and 2013Z007), high-level topic-matching funds from 


\section{Cellular Physiology Cell Physiol Biochem 2018;47:590-603 \begin{tabular}{l|l} 
DOI: 10.1159/000490015 & and Biochemistry \\
Published online:TMay 25, 2018 & $\begin{array}{l}\text { O 2018 The Author(s). Published by S. Karger AG, Basel } \\
\text { www.karger.com/cpb }\end{array}$
\end{tabular}}

Huang et al: Molecular Regulation of CRC Metastasis

Nanfang Hospital (Nos.201347 and G201227),The funding organization: Guangdong Provincial Key Laboratory of Gastroenterology, Department of Gastroenterology, Nanfang Hospital, Southern Medical University, and the Guangzhou Pilot Project of the Clinical and Translational Research Center (early gastrointestinal cancer, No.7415696196402).

\section{Disclosure Statement}

The authors declare that they have no competing interests.

\section{References}

1 Kalluri R, Neilson EG: Epithelial-mesenchymal transition and its implications for fibrosis. J Clin Invest 2003;112:1776-1784.

2 Carew RM, Wang B, Kantharidis P: The role of EMT in renal fibrosis.Cell Tissue Res 2012;347:103-116.

3 Ono H, Imoto I, Kozaki K, Tsuda H, Matsui T, Kurasawa Y, Muramatsu T, Sugihara K, Inazawa J: SIX1 promotes epithelial-mesenchymal transition in colorectal cancer through ZEB1 activation. Oncogene 2012;31:49234934.

-4 Xu W, Wang Z, Zhang W, Qian K, Li H, Kong D, Li Y, Tang Y: Mutated K-ras activates CDK8 to stimulate the epithelial-to-mesenchymal transition in pancreatic cancer in part via the $\mathrm{Wnt} / \beta$-catenin signaling pathway. Cancer Lett 2015;356:613-627.

5 Liu CC, Cai DL, Sun F, Wu ZH, Yue B, Zhao SL, Wu XS, Zhang M, Zhu XW, Peng ZH, Yan DW: FERMT1 mediates epithelial-mesenchymal transition to promote colon cancer metastasis via modulation of $\beta$-catenin transcriptional activity. Oncogene 2017;36:1779-1792.

6 Oh BY, Kim SY, Lee YS, Hong HK, Kim TW, Kim SH, Lee WY, Cho YB: Twist1-induced epithelial-mesenchymal transition according to microsatellite instability status in colon cancer cells. Oncotarget 2016;7:5706657076.

7 Kroepil F, Fluegen G, Totikov Z, Baldus SE, Vay C, Schauer M, Topp SA, Esch JS, Knoefel WT, Stoecklein NH: Down-regulation of CDH1 is associated with expression of SNAI1 in colorectal adenomas. PLoS One 2012;7:e46665.

8 Larriba MJ, Martín-Villar E, García JM, Pereira F, Peña C, de Herreros AG, Bonilla F, Muñoz A: Snail2 cooperates with Snail1 in the repression of vitamin D receptor in colon cancer. Carcinogenesis 2009;30:1459-1468.

-9 Korpal M, Lee ES, Hu G, Kang Y: The miR-200 family inhibits epithelial- mesenchymal transition and cancer cell migration by direct targeting of E-cadherin transcriptional repressors ZEB1 and ZEB2. J Biol Chem 2008;283:14910-14914.

10 Huang JZ, Chen M, Zeng M, Xu SH, Zou FY, Chen D, Yan GR: Down-regulation of TRPS1 stimulates epithelial-mesenchymal transition and metastasis through repression of FOXA1. J Pathol 2016;239:186196.

11 Chuang WJ, Yeh IJ, Hsieh YH, Liu PP, Chen SW, Jeng WY: 1H, 15N and 13C resonance assignments for the DNA-binding domain of myocyte nuclear factor (Foxk1). J Biomol NMR 2002;24:75-76.

12 Wu Y, Peng Y, Wu M, Zhang W, Zhang M, Xie R, Zhang P, Bai Y, Zhao J, Li A, Nan Q, Chen Y, Ren Y, Liu S, Wang J: Oncogene FOXK1 enhances invasion of colorectal carcinoma by inducing epithelial-mesenchymal transition. Oncotarget 2016;7:51150-51162.

13 Xie R, Wang J, Liu X, Wu L, Zhang H, Tang W, Li Y, Xiang L, Peng Y, Huang X, Bai Y, Liu G, Li A, Wang Y, Chen Y, Ren Y, Li G, Gong W, Liu S, Wang J: RUFY3 interaction with FOXK1 promotes invasion and metastasis in colorectal cancer. Sci Rep 2017;7:3709.

14 Wang W, Li X, Lee M, Jun S, Aziz KE, Feng L, Tran MK, Li N, McCrea PD, Park JI, Chen J: FOXKs promote Wnt/ $\beta$ catenin signaling by translocating DVL into the nucleus. Dev Cell 2015;32:707-718.

15 Peng Y, Zhang P, Huang X, Yan Q Wu M, Xie R, Wu Y, Zhang M, Nan Q Zhao J, Li A, Xiong J, Ren Y, Yang B, Chen Y, Liu S, Wang J: Direct regulation of FOXK1 by c-jun promotes proliferation, invasion and metastasis in gastric cancer cells. Cell Death Dis 2016;7:e2480.

16 Zhou BP, Deng J, Xia W, Xu J, Li YM, Gunduz M, Hung MC: Dual regulation of Snail by GSK-3beta-mediated phosphorylation in control of epithelial-mesenchymal transition. Nat Cell Biol 2004;6:931-940.

-17 Blanco MJ, Moreno-Bueno G, Sarrio D, Locascio A, Cano A, Palacios J, Nieto MA: Correlation of Snail expression with histological grade and lymph node status in breast carcinomas. Oncogene 2002;21:3241-3246. 


\section{Cellular Physiology Cell Physiol Biochem 2018;47:590-603

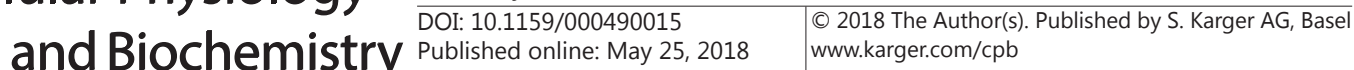

Huang et al: Molecular Regulation of CRC Metastasis

18 Zhang W, Wang J, Zou B, Sardet C, Li J, Lam CS, Ng L, Pang R, Hung IF, Tan VP, Jiang B, Wong BC: Four and a half LIM protein 2 (FHL2) negatively regulates the transcription of E-cadherin through interaction with Snail1. Eur J Cancer 2011;47:121-130.

19 Saglam O, Dai F, Husain S, Zhan Y, Toruner G, Haines GK 3rd: Matricellular protein CCN1 (CYR61) expression is associated with high-grade ductal carcinoma in situ. Hum Pathol 2014;45:1269-1275.

20 Chang CC, Hsu WH, Wang CC, Chou CH, Kuo MY, Lin BR, Chen ST, Tai SK, Kuo ML, Yang MH: Connective tissue growth factor activates pluripotency genes and mesenchymal-epithelial transition in head and neck cancer cells. Cancer Res 2013;73:4147-4157.

21 Cui L, Xie R, Dang S, Zhang Q Mao S, Chen J, Qu J, Zhang J: NOV promoted the growth and migration of pancreatic cancer cells. Tumour Biol 2014;35:3195-3201.

22 Cardoso J, Mesquita M, Dias Pereira A, Bettencourt-Dias M, Chaves P, Pereira-Leal JB: CYR61 and TAZ Upregulation and Focal Epithelial to Mesenchymal Transition May Be Early Predictors of Barrett's Esophagus Malignant Progression. PLoS One 2016;11:e0161967.

23 Jeong D, Heo S, Sung Ahn T, Lee S, Park S, Kim H, Park D, Byung Bae S, Lee SS, Soo Lee M, Kim CJ, Jun Baek M: Cyr61 expression is associated with prognosis in patients with colorectal cancer. BMC Cancer 2014;14:164.

-24 Hou CH, Lin FL, Hou SM, Liu JF: Cyr61 promotes epithelial-mesenchymal transition and tumor metastasis of osteosarcoma by Raf-1/MEK/ERK/Elk-1/TWIST-1 signaling pathway. Mol Cancer 2014;13:236.

25 Chin LH, Hsu SP, Zhong WB, Liang YC: Involvement of cysteine-rich protein 61 in the epidermal growth factor-induced migration of human anaplastic thyroid cancer cells. Mol Carcinog 2016;55:622-632.

26 Wu M, Wang J, Tang W, Zhan X, Li Y, Peng Y, Huang X, Bai Y, Zhao J, Li A, Chen C, Chen Y, Peng H, Ren Y, Li G, Liu S, Wang J: FOXK1 interaction with FHL2 promotes proliferation, invasion and metastasis in colorectal cancer. Oncogenesis 2016;5:e271.

27 Wang G, Gu J, Gao Y: MicroRNA target for MACC1 and CYR61 to inhibit tumor growth in mice with colorectal cancer. Tumour Biol 2016;37:13983-13993.

28 Liu R, Huang S, Lei Y, Zhang T, Wang K, Liu B, Nice EC, Xiang R, Xie K, Li J, Huang C: FGF8 promotes colorectal cancer growth and metastasis by activating YAP1. Oncotarget 2015;6:935-952.

-29 Yang Q Bassel-Duby R, Williams RS: Transient expression of a winged-helix protein, MNF-beta, during myogenesis. Mol Cell Biol 1997;17:5236-5243.

30 Mistry DS, Chen Y, Wang Y, Sen GL: Transcriptional profiling of SNAI2 regulated genes in primary human keratinocytes. Genom Data 2015;4:43-46.

-31 Bowman CJ, Ayer DE, Dynlacht BD: Foxk proteins repress the initiation of starvation-induced atrophy and autophagy programs. Nat Cell Biol 2014;16:1202-1214.

-32 Li P, Yu Z, He L, Zhou D, Xie S, Hou H, Geng X: Knockdown of FOXK1 inhibited the proliferation, migration and invasion in hepatocellular carcinoma cells. Biomed Pharmacother 2017;92:270-276.

33 Chen F, Xiong W, Dou K, Ran Q: Knockdown of FOXK1 Suppresses Proliferation, Migration, and Invasion in Prostate Cancer Cells. Oncol Res 2017;25:1261-1267.

-34 Zhang P, Tang WM, Zhang H, Li YQ Peng Y, Wang J, Liu GN, Huang XT, Zhao JJ, Li G, Li AM, Bai Y, Chen Y, Ren YX, Li GX, Wang YD, Liu SD, Wang JD: MiR-646 inhibited cell proliferation and EMT-induced metastasis by targeting FOXK1 in gastric cancer. Br J Cancer 2017, 117:525-534.

-35 Castro Alves C, Rosivatz E, Schott C, Hollweck R, Becker I, Sarbia M, Carneiro F, Becker KF: Slug is overexpressed in gastric carcinomas and may act synergistically with SIP1 and Snail in the down-regulation of E-cadherin. J Pathol 2007;211:507-515.

36 Yook JI, Li XY, Ota I, Fearon ER, Weiss SJ: Wnt-dependent regulation of the E-cadherin repressor snail. J Biol Chem 2005;280:11740-11748.

37 Leng E, Malcolm T, Tai G, Estable M, Sadowski I: Organization and expression of the Cyr61 gene in normal human fibroblasts. J Biomed Sci 2002;9:59-67.

-38 Wu G, Zhu YZ, Zhang JC: Sox4 up-regulates Cyr61 expression in colon cancer cells. Cell Physiol Biochem 2014;34:405-412.

-39 Kroepil F, Fluegen G, Vallböhmer D, Baldus SE, Dizdar L, Raffel AM, Hafner D, Stoecklein NH, Knoefel WT: Snail1 expression in colorectal cancer and its correlation with clinical and pathological parameters.BMC Cancer 2013;13:145.

-40 Kwon CH, Park HJ, Choi JH, Lee JR, Kim HK, Jo HJ, Kim HS, Oh N, Song GA,Park DY: Snail and serpinA1 promote tumor progression and predict prognosis in colorectal cancer. Oncotarget 2015;6:20312-20326. 